\title{
Mise en service des dessertes TGV et gouvernance dans le domaine du tourisme : le cas de villes françaises
}

Opening of High-speed rail Services and tourism governance: a focus on French cities

Inbetriebnahme einer TGV-Verkehrsanbindung und "Gouvernance" im

Tourismusbereich : anhand einer Verdeutlichung französischer Städte

Sylvie Bazin-Benoit and Marie Delaplace

\section{(2) OpenEdition}

\section{Journals}

Electronic version

URL: http://journals.openedition.org/rge/5614

ISSN: 2108-6478

Publisher

Association des géographes de l'Est

Printed version

Date of publication: 1 October 2015

ISSN: 0035-3213

Electronic reference

Sylvie Bazin-Benoit and Marie Delaplace, "Mise en service des dessertes TGV et gouvernance dans le domaine du tourisme : le cas de villes françaises », Revue Géographique de l'Est [Online], vol.55 / n³-4 | 2015, Online since 17 October 2015, connection on 08 September 2020. URL : http:// journals.openedition.org/rge/5614

This text was automatically generated on 8 September 2020

Tous droits réservés 


\section{Mise en service des dessertes TGV et gouvernance dans le domaine du tourisme : le cas de villes françaises}

Opening of High-speed rail Services and tourism governance: a focus on French cities

Inbetriebnahme einer TGV-Verkehrsanbindung und "Gouvernance" im Tourismusbereich : anhand einer Verdeutlichung französischer Städte

Sylvie Bazin-Benoit and Marie Delaplace

\section{Introduction}

1 La mise en service d'une desserte TGV (i.e. une desserte en TGV en totalité ou en partie sur ligne à grande vitesse) est souvent porteuse d'espoirs pour les territoires desservis, notamment s'ils souffrent de difficultés économiques. Celle-ci peut en effet être perçue comme une opportunité au service d'une attractivité renouvelée. Des formes de coordination nouvelles, entre territoires et acteurs concurrents ou aux intérêts divergents (collectivités locales, opérateurs ferroviaires, entreprises) peuvent alors émerger. Certaines de ces coordinations innovantes sont reconnues aujourd'hui comme ayant bénéficié aux territoires desservis en termes d'attractivité en général.

2 Mais la question de la gouvernance associée aux dessertes TGV en lien avec le tourisme a peu été abordée. C'est pourtant un des domaines dans lesquels les espoirs de valorisation des dessertes TGV sont importants, même s'ils ne se réalisent pas toujours, et la question de la gouvernance, fondamentale. En effet, une destination touristique incorporant de nombreux produits et services sur un territoire donné (hébergements, restaurations, aménités diverses de nature culturelle, naturelle ou patrimoniale, services de transport, etc.) la production de cette destination exige que les acteurs (publics et privés du tourisme au sens large, de la culture et du transport, offices du tourisme, collectivités, prestataires privés du tourisme, musées, SNCF, gestionnaire de 
la gare, etc.) qui participent de la réalisation de ces différents produits et services se coordonnent. L'arrivée d'une desserte TGV les invite à le faire pour tirer parti de la meilleure accessibilité de leur territoire.

Des formes de gouvernance nouvelles peuvent alors apparaitre à différentes échelles territoriales et entre différents types d'acteurs, se traduisant par une communication commune, une offre de tarifs préférentiels ciblés sur des opérations spécifiques, telles des festivals, des "offres packagées", ou un élargissement de la destination en associant à la destination principale une ou des destinations secondaires. Une cinquantaine d'entretiens réalisés avec des acteurs locaux dans différents types de villes : grandes (Lille et Nantes), intermédiaires (Le Mans, Metz, Reims et Tours) et petites et moyennes (Auray, Arras, Charleville-Mézières, Saverne et Vendôme) nous permettent d'identifier ces formes de gouvernance et leur efficacité.

Après avoir établi le lien possible entre grande vitesse ferroviaire et gouvernance locale dans le domaine du tourisme (I), l'analyse de ces villes nous permet d'identifier les outils de gouvernance mis en place dans ce secteur en lien avec les dessertes TGV (II).

\section{Gouvernance locale, grande vitesse ferroviaire et tourisme : quel lien établir?}

5 Sur un territoire se mettent en place des processus d'interaction et de collaboration entre acteurs de nature hétérogène, et poursuivant des objectifs parfois différents (Leloup, Moyart, Pecqueur, 2005). Une gouvernance est alors nécessaire. Celle-ci renvoie au pilotage de logiques interdépendantes, de nature politique, juridique, géographique, sociologique, économique, voire environnementale, (Jacquier, 2008). La mise en service de dessertes ferroviaires à grande vitesse peut modifier les modalités de la gouvernance et engendrer de nouvelles formes de coordination (A). C'est le cas particulièrement pour le tourisme, activité pour laquelle la question de la coordination se pose de façon importante, et en particulier les types de tourisme valorisables par les dessertes TGV (B).

\section{A. Dessertes TGV et gouvernance locale: de nombreux domaines concernés}

6 La question de la gouvernance se pose lors de la construction de la ligne à grande vitesse (LGV) mais aussi au moment voire après la mise en service de la desserte.

7 Préalablement à la mise en service de l'infrastructure, la gouvernance est impactée par l'ensemble des réflexions et décisions issues de la concertation entre acteurs pour la définition de la nouvelle desserte, la localisation des gares, et les politiques d'accompagnement à mettre en place. La question de la localisation des gares nouvelles sur LGV, se pose très en amont lors de la définition du tracé. Les conflits entre territoires concernés peuvent alors être vifs, chacun souhaitant obtenir une gare au plus proche de sa ville dans la mesure où les dessertes TGV sont souvent perçues comme une opportunité de développement local.

8 La définition de la nouvelle desserte nécessite aussi une concertation entre l'opérateur ferroviaire et les villes concernées, les associations d'usagers, et les financeurs quant aux horaires et à la fréquence. Cette concertation est supposée faire émerger un 
consensus sur les effets indirects et collatéraux de la nouvelle ligne et le réaménagement nécessaire des dessertes secondaires impactées. Les discussions préalables aux compromis font ressortir une lutte d'intérêts et un positionnement spécifique de chaque acteur compte tenu de ses attentes vis-à-vis de la nouvelle infrastructure. Ces réunions de concertation modifient les rapports entre acteurs et contribuent à la redéfinition de leur positionnement dans les rapports de force existant.

C'est également à ce moment-là que les acteurs publics cherchent à mettre en place des observatoires visant à mesurer les impacts potentiels liés aux dessertes. Ces observatoires qui rassemblent tous types d'acteurs concernés de près ou de loin par les dessertes TGV (agences d'urbanisme, villes, universités, représentants de l'Etat, etc.) nécessitent alors de nouveaux modes de coordination.

Une fois les compromis établis, et toujours préalablement à la mise en service des dessertes, les acteurs du territoire s'interrogent sur leur potentiel de valorisation dans des domaines divers (immobilier résidentiel et d'entreprises, intermodalité, économique, universitaire, touristique, etc.) selon les caractéristiques des territoires. Cela donne lieu à des études prospectives qui à partir de réflexions sur le devenir du territoire débouchent sur des projets et la mise en place de politiques d'accompagnement.

11 Ces politiques s'articulent généralement autour de trois domaines: l'attractivité, la mobilité, et les politiques de communication.

12 1. En termes d'attractivité, les acteurs locaux considèrent que les dessertes TGV faciliteront la venue d'entreprises, de ménages, d'étudiants, voire de touristes dans le cas de villes présentant un potentiel dans ce domaine. Des politiques de construction de centres d'affaire autour des gares, de zones d'habitat nouvelles et de zones d'activité à proximité des quartiers des gares sont alors mises en œuvre et différents acteurs sont amenés à y participer. Dans le domaine de l'immobilier d'entreprise et résidentiel, par exemple, interviennent ainsi des communes ou intercommunalités, des propriétaires des réserves foncières, des promoteurs immobiliers, des bailleurs sociaux, des associations de défense du patrimoine (en cas de démolition de bâtiments anciens), voire des habitants.

13 2. En termes de mobilité, outre la possible induction de trafic, les acteurs s'attendent à des reports modaux de la route vers le rail (TGV, ou même le TER dans le cas de combinaisons de trajets TER/TGV). Les politiques visent alors à améliorer l'intermodalité, à mettre en place des dessertes intra-urbaines plus adaptées à la future demande, voire d'en créer de nouvelles (tramways, station de taxis ou vélibs), des dessertes entre gares centres et gares TGV, le cas échéant. Un aménagement de pôles intermodaux autour des gares peut être prévu. Dans ce domaine, ce sont les villes, les prestataires privés comme les taxis, les opérateurs des transports urbains comme les régies ou autres opérateurs participant à des PPP, les associations d'usagers des transports, les architectes susceptibles d'intégrer à leurs projets ces nouvelles configurations intermodales, qui doivent se coordonner autour de ces projets.

14 3. En termes de communication, la mise en service d'une LGV constitue une opportunité que la plupart des territoires saisissent en multipliant les opérations, préalablement, au moment et postérieurement à la mise en service. Les formes de communication diverses visent des cibles régionales, nationales ou internationales (recherche d'investisseurs extérieurs par des associations de promotion du territoire; 
promotion à l'étranger par le biais d'agences de développement). Ainsi en France, la grande vitesse est utilisée en termes d'image (cf. Carrouet, 2013, pour ce qui concerne la LGV Rhin-Rhône; ou ISIS, 2004 pour la LGV Est-européenne et les villes de Metz, Nancy, Epinal et Thionville). La recherche d'une image est également associée à un effet «club " pour les villes desservies. Lors de la mise en service des dernières dessertes, la SNCF a créé des clubs TGV auxquels adhèrent de nombreuses collectivités à différentes échelles (agglomérations, départements ou régions) qui les utilisent pour communiquer mais aussi des groupements d'entreprises. Ces politiques de communication participent à l'évolution de la gouvernance territoriale au sens où en cherchant à modifier l'image véhiculée par le territoire à l'extérieur, les acteurs modifient également l'image qu'ils en ont eux-mêmes. Ceci contribue à renforcer la confiance et l'ambition qu'ils peuvent avoir pour leur territoire et à bâtir des politiques associées à cette ambition.

En outre, au moment de la mise en service de la desserte, des évènements qui mobilisent acteurs publics et privés sont souvent organisés; les retombées commerciales attendues étant importantes. Des opérations ponctuelles peuvent être proposées par l'opérateur, telles que des billets promotionnels.

Après la mise en service de la ligne, un certain nombre de politiques se poursuivent, notamment concernant la communication ou l'aménagement des abords des gares.

17 Ces réflexions, décisions et politiques suscitées par la mise en service des lignes modifient le positionnement des acteurs et renouvelle de fait le mode de gouvernance $\mathrm{du}$ territoire particulièrement dans le domaine du tourisme.

\section{B. Des dessertes TGV au cœur de la coordination pour certains types de tourisme}

Le tourisme est une activité qui incorpore des services différents : l'hébergement, la restauration, les activités de loisirs, les visites de sites, la dégustation de spécialités locales, etc.. C'est l'ensemble de ces activités qui forme la destination touristique que le touriste recherche et apprécie. Ces différentes activités doivent ainsi être coordonnées au sein de la destination. Dans certains cas elles peuvent être internalisées dans une même entreprise; c'est le cas des "resorts", complexes touristiques qui sont des formes de tourisme hors sol. Mais ces activités sont aussi souvent produites par différentes entreprises (hôteliers, restaurants, musées, parc d'attractions, producteurs locaux, etc.) et doivent alors être coordonnées. Si leur coordination peut être effectuée par le biais de relations marchandes (d'achat-vente), ce n'est pas le cas de toutes, notamment lorsqu'elles mobilisent des ressources apparentées à des biens publics. Par exemple, le paysage peut être considéré comme une ressource produite et entretenue collectivement par des acteurs sur un territoire. Sa conservation passe par l'action conjointe de tous les acteurs sur ce territoire.

19 En outre, le caractère pérenne d'une destination touristique repose sur la durabilité de la coordination. Il s'agit d'éviter les comportements opportunistes, visant uniquement à vendre un produit ou un service sans se préoccuper de sa qualité. Le touriste est en effet susceptible de revenir mais aussi de faire venir d'autres touristes.

Par ailleurs le tourisme présente la caractéristique d'impliquer des acteurs publics (office du tourisme, comité départemental du tourisme, etc.) et privés ou associatifs (associations de sauvegarde de site, associations sportives, prestataires, etc.). Le 
caractère fragmenté de l'industrie du tourisme nécessite alors une coordination et une collaboration importante entre les différentes parties-prenantes pour assurer le succès de la destination (Wang and. Fesenmaier, 2007, Jones and al. 2013). Cette coopération entre acteurs privés et publics à l'intérieur d'un même secteur (la culture) ou entre secteurs différents (la culture et la restauration) est ainsi une des conditions qui permet à une région touristique de dégager un avantage compétitif (Czernek, 2013).

Or le transport qui est intrinsèquement lié au comportement du touriste, par définition en déplacement. est un facteur important de développement du tourisme (Prideaux, 2000). Les acteurs du transport vers et dans la destination peuvent alors être une des parties-prenantes de cette coopération, notamment au moment de l'arrivée d'une desserte TGV.

Les territoires proposant des actifs spécifiques (Colletis et Pecqueur, 2004), quelle que soit leur nature (patrimoniale, mémorielle, balnéaire, culturelle, etc.), peuvent ainsi tenter de les valoriser avec une desserte TGV, les tourismes urbain et d'affaire étant principalement concernés.

Les tourismes urbains (visites de musées, monuments historiques, dégustations gastronomiques, shopping) et d'affaire (séminaires, incentives, etc.) sont les tourismes les plus à même d'être valorisés par une desserte à grande vitesse (Delaplace et Perrin, 2013 ; Kamel et Matthewman, 2008). Il s'agit de tourismes de court séjour (deux ou trois jours) et souvent de fin de semaine, pratiqués principalement par des personnes seules ou des couples appartenant à des professions et catégories socioprofessionnelles diversifiées. La desserte TGV, si elle est de qualité, permet d'être plus rapidement sur place et sans fatigue.

Dès lors que les aménités sont localisées au cœur des villes desservies et près de la gare centrale ou qu'une gestion de l'acheminement vers les lieux touristiques est proposée, tous les tourismes s'inscrivant dans les villes peuvent bénéficier des dessertes TGV. C'est le cas du tourisme évènementiel.

Le tourisme d'affaires est quant à lui un tourisme effectué dans le cadre de l'activité professionnelle. Il comprend les congrès, conférences, colloques ou symposium, séminaires ou workshop, ou encore des visites de salons, de foires, et des incentives (réunions dont l'objectif est de motiver les employés d'une organisation). Il est caractérisé par des déplacements individuels et/ou collectifs de court séjour en semaine décidés par les entreprises ou les administrations avec une forte importance accordée à la réduction des temps de parcours. Comme pour d'autres formes de tourisme, une tendance au raccourcissement de la durée moyenne des séjours peut être notée (Coach Omnium, 2014). Les villes TGV sont ainsi clairement convoitées par les acteurs du tourisme d'affaires (Amiard, 1997), l'accessibilité étant un des premiers critères de choix d'une destination (De Seze, 2008).

Les tourismes situés en dehors des villes (par exemple, vert ou de montagne) peuvent plus difficilement bénéficier de la desserte; excepté si l"intermodalité qui permet au touriste de rejoindre sa destination sans perte de temps importante a été pensée. Cette gestion de l'intermodalité se traduit par la mise en place de location de vélos (parfois gratuitement dans le cadre de packages) en gare, des navettes desservant des points d'accès aux sites, des liaisons TER facilitant l'accès à des destinations touristiques secondaires, villes ou musées, etc.. Ces politiques peuvent alors favoriser le 
développement des fréquentations touristiques de sites proches des villes principalement desservies.

La valorisation de ces types de tourisme par des politiques d'accompagnement (Bazin et al., 2010) se traduit par des politiques de communication, d'intermodalité, et d'offre renouvelée de produits touristiques.

En termes de communication, les acteurs peuvent s'emparer des dessertes TGV pour modifier l'image de leur territoire. Les dessertes TGV sont ainsi à l'origine d'un effet de notoriété, d'un signe de distinction dont bénéficient les territoires desservis et en particulier le tourisme (Mignerey, 2013, Setec Organisation, 2005). Cela a été le cas au Mans, à Lille, Arras et Reims (Bazin et al, 2013a et 2014 ; Menerault, 1997), ou encore dans les villes desservies par la LGV Rhin-Rhône (Carrouet, 2013). Les acteurs du tourisme aux échelles locale, départementale ou régionale cherchent alors à valoriser les dessertes TGV dans leur politique de communication.

Les opérateurs ferroviaires peuvent collaborer avec certaines villes de façon à valoriser l'évènementiel et rentabiliser la ligne les week-ends. En coopération avec la SNCF, les villes desservies ont souvent utilisé la desserte pour faire parler d'elles et inversement. Des évènements festifs sont également organisés au moment de l'ouverture des lignes et parfois des billets à tarif préférentiel sont proposés. Ils concernent toutefois généralement les plus grandes villes et les évènements les plus connus. L'opérateur ferroviaire apparait alors souvent maittre du jeu des politiques de valorisation de la ligne à des fins de fréquentation évènementielle ou touristique. Ainsi, lors de la mise en service du TGV Est-Européen, la SNCF a vendu des billets à prix réduit, ce qui a conduit momentanément à développer le tourisme dans les villes nouvellement desservies. L'opérateur ferroviaire peut aussi proposer pour certaines destinations une offre de transport supplémentaire lors d'évènements exceptionnels. Ainsi, à Strasbourg des TGV sont affrétés pour le marché de Noël. A Lille au moment de la grande braderie, la capacité de certains TGV entre Lille et Paris est augmentée. A Belfort, lors des Eurockéennes, deux TGV 100\% Prem's ont été mis en place (site des Eurockéennes). Les acteurs du territoire s'associent et impulsent des campagnes de communication qui leur permettent de s'approprier l'image associée au TGV. Mais des conflits en termes de communication peuvent brouiller l'image de la ville. Des communications peuvent en effet être contradictoires. Le dynamisme de la construction atteste sans doute du dynamisme d'une ville mais n'incite pas à venir la visiter. 
Figure 1 : Invest in Reims, Reims une ville qui construit

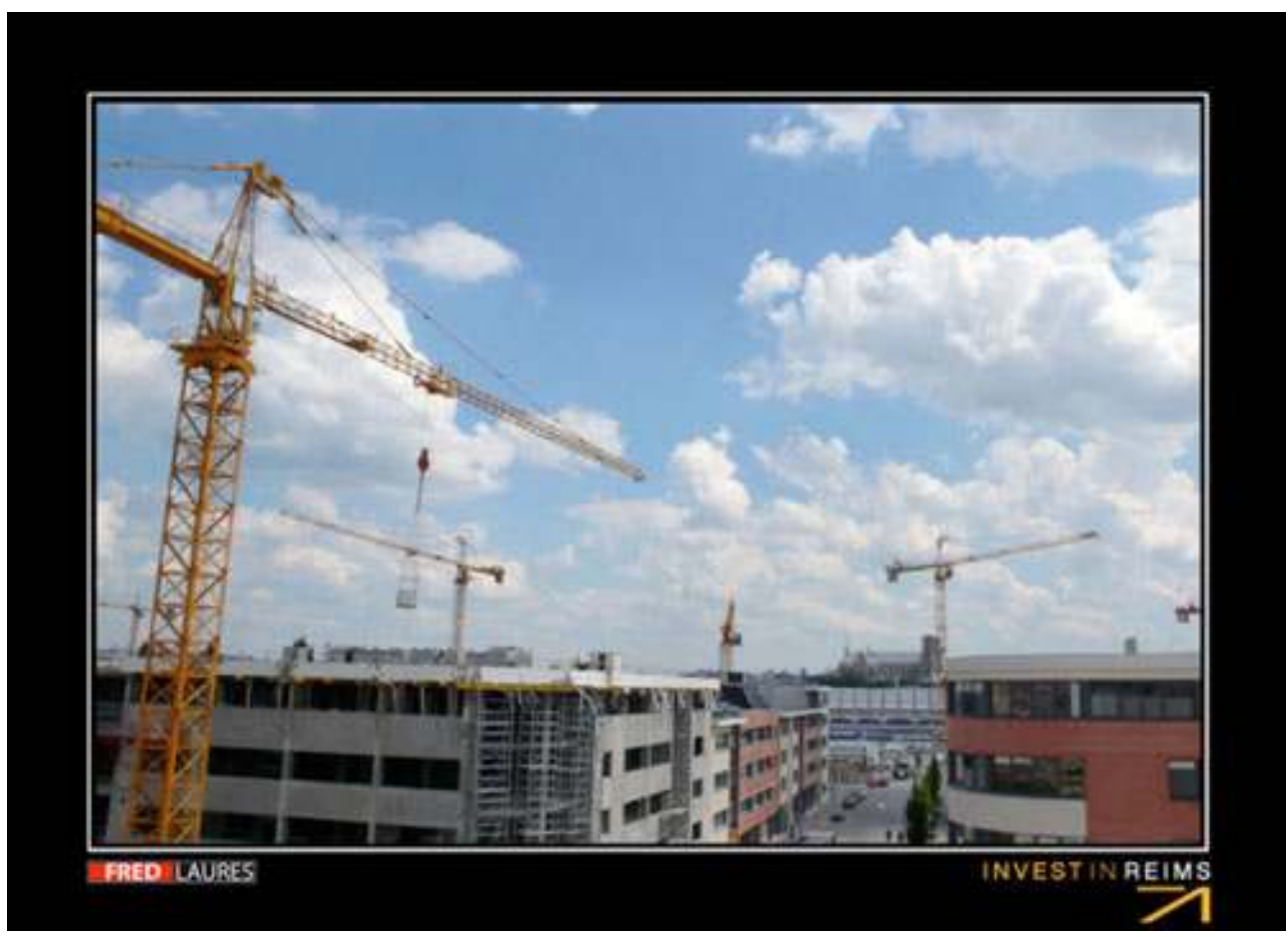

Figure 2 : Office du tourisme Reims, 2000 ans d'histoire

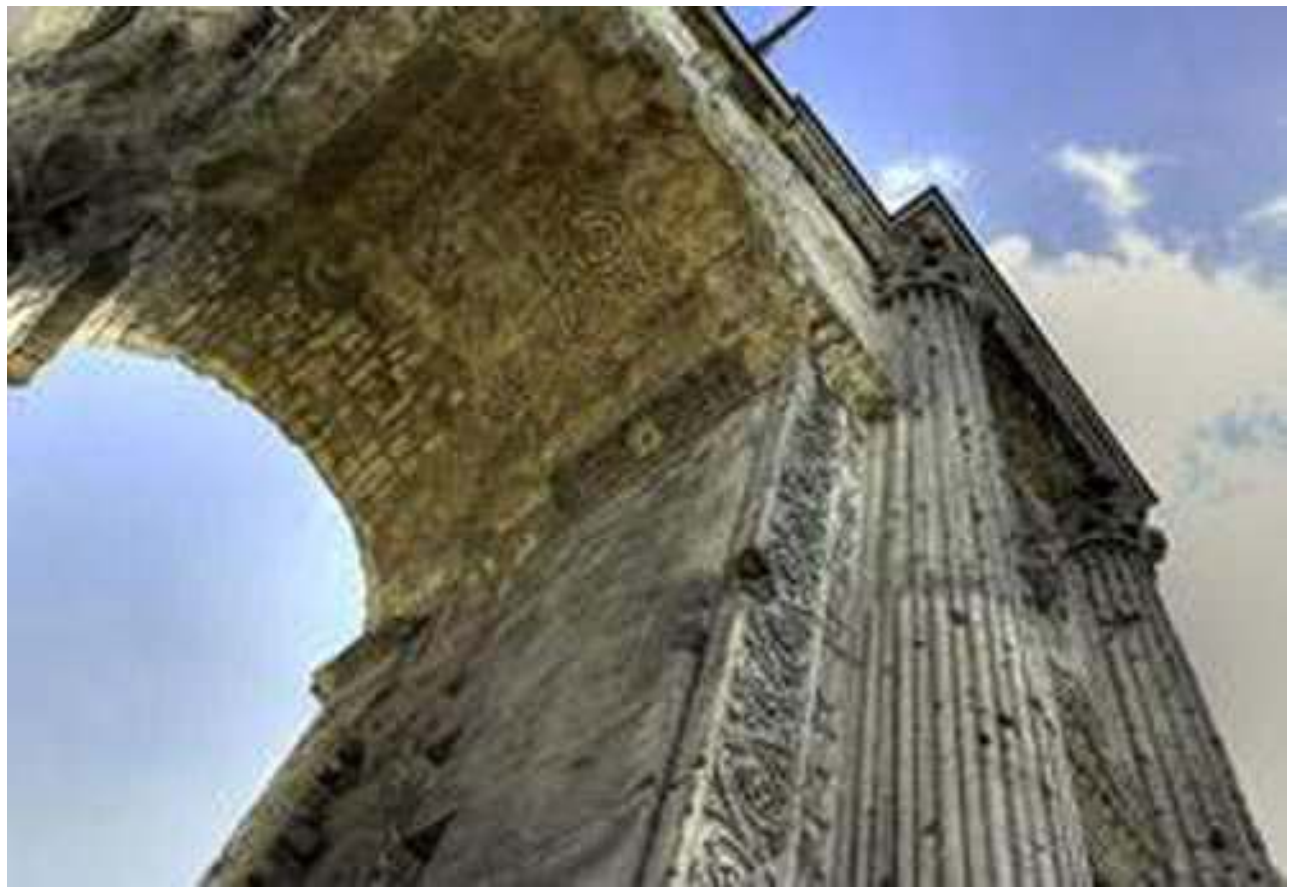

La coordination des différents acteurs dans la promotion de la destination doit alors être assurée et la gouvernance devient un enjeu central.

Dès lors que leur activité peut être impactée par le TGV, des acteurs privés peuvent également envisager de se coordonner avec les acteurs publics ou parapublics en termes de communication. C'est le cas des palais de Congrès dont la fréquentation peut bénéficier des dessertes TGV (Delaplace, Perrin, 2013), des clubs hôteliers ou 
d'entreprises qui souhaitent être associés d'un point de vue marketing à leur territoire d'implantation.

Par ailleurs, en termes d'intermodalité, si la desserte TGV permet d'arriver plus vite, le temps gagné pendant le voyage ne doit pas être perdu à l'arrivée par une insuffisante articulation avec les transports locaux. La nouvelle desserte est donc fréquemment accompagnée par une réflexion concernant les interconnexions en termes de transport (TER, transports urbains collectifs, navettes spéciales, voire cheminements pédestres (Coronado et al., 2014) pour amener les touristes à leur destination finale. La collectivité locale peut redéfinir l'insertion urbaine de la gare qui constitue une des portes de la ville. A Reims, l'organisation des transports urbains a été repensée à partir de la gare centre. En revanche cela n'a pas été fait à Tours, où l'arrivée des TGV et le départ des circuits touristiques ne sont pas articulés (Troin, 2012).

Enfin, un des principaux enjeux associés à la desserte est de lutter contre la réduction de la durée des séjours. Un travail sur les produits proposés par la destination en renforçant le panier de biens et services territorialisés (Pecqueur, 2001; Mollard et Pecqueur, 2007) est souvent effectué. Le touriste peut alors y puiser pour construire son séjour. Des produits nocturnes (illuminations, visites nocturnes, etc.) contribuant à renouveler l'offre de produits touristiques peuvent être développés de manière à ce que le touriste soit incité à rester la nuit. Mais pour que le tourisme se développe, il est aussi nécessaire que les capacités hôtelières soient suffisantes et de surcroît adaptées aux types de touristes ciblés. Ainsi le développement du tourisme d'affaires ne peut se produire sans capacités hôtelières importantes en quantité et qualité. Il est alors nécessaire de penser un renouvellement de l'offre d'hébergement.

L'ensemble de ces politiques nécessite une coordination entre acteurs publics et privés de la destination (agence de voyages, organisateur d'évènements culturels, office du tourisme, SNCF, prestataire de transports urbains, hôteliers, restaurateurs, etc.). Ceuxci produisent des packages incorporant un billet TGV, un hébergement, et/ou une visite de musée et/ou un concert, un Pass pour visiter la ville, etc.. Dans certains cas, les offices de tourisme des territoires proches proposent des circuits incitant les touristes à visiter d'autres lieux (Bazin et al., 2011b).

En matière de tourisme, les modes de gouvernance sont ainsi amenés à évoluer, en lien avec la mise en service de dessertes TGV. Mais les possibilités de valorisation dépendent des caractéristiques des villes. Leur taille conditionne ainsi les politiques d'accompagnement, les plus grandes villes disposant de moyens plus importants. Leur localisation joue également un rôle important. Les attentes liées aux dessertes TGV sont en effet différentes selon que les villes sont centrales ou périphériques, les dessertes TGV bénéficiant généralement davantage à la centralité (Bazin et al, 2011a). Toutefois le fait d'être une ville de petite taille, périphérique, mais en façade maritime par exemple, peut expliquer des attentes importantes et des politiques associées. Les territoires qui présentent des aménités climatiques ou géographiques spécifiques anticipent également une utilisation du TGV pour motif de tourisme. 


\section{Gouvernance locale, tourisme et dessertes TGV : le cas de trois types de villes françaises}

Le tourisme et particulièrement le tourisme urbain et d'affaires peuvent être impactés par la mise en service d'une desserte TGV dès lors que les villes sont dotées d'actifs spécifiques (A). Par la mise en œuvre de politiques adéquates, le type de gouvernance locale qui émerge peut permettre de valoriser la desserte à des fins touristiques, comme le montre l'analyse de différentes villes françaises (B).

\section{A. Types de villes concernées par la valorisation touristique de leur desserte TGV}

La taille des villes desservies influe de façon évidente sur le potentiel de valorisation des dessertes. Le TGV valorise en effet des lieux déjà connus, bénéficiant d'une certaine notoriété, et disposant d'une offre de ressources spécifiques ${ }^{1}$ suffisamment importante pour attirer les touristes. Cette offre qui peut être liée à un patrimoine (historique, culturel, gastronomique) ou à un évènement dépend généralement de la taille des villes. Cette dernière est également déterminante dans l'existence d'une capacité hôtelière et de restauration.

Les capitales sont ainsi souvent convoitées en matière de tourisme par la clientèle étrangère, même si celle-ci apprécie également une visite "secondaire " en province lors d'un séjour, le choix se portant généralement sur une autre grande ville. Ainsi Pagliara et al., 2012, montrent que la desserte TGV influe sur la probabilité des touristes de visiter des villes reliées par TGV à Rome. Il en va de même à Madrid (Pagliara et al., 2014). La clientèle nationale elle, effectue un arbitrage entre des villes de taille intermédiaire, ce qui conduit à une hiérarchie de choix visant secondairement la visite d'une petite ville, dès lors que celle-ci propose également des aménités spécifiques.

Dans la mesure où les conditions d'accès peuvent être modifiées après la mise en service d'une desserte TGV, des villes ne faisant jusqu'alors pas partie de la "short list » peuvent y être désormais intégrées. Inversement des villes non desservies peuvent en sortir. Dès lors que la fréquence est de qualité et la grille horaire adéquate pour des déplacements touristiques, la desserte peut faire évoluer le classement des villes par la clientèle touristique. Mais, une ville bien desservie au moment de la mise en service d'une ligne peut voir la qualité de sa desserte progressivement se dégrader suite à une évolution ultérieure de l'offre ferroviaire, fragilisant alors l'activité touristique. Par ailleurs, le nombre de villes TGV ayant cru de façon importante, le fait de disposer d'une desserte n'est un avantage que si les actifs spécifiques sont jugés suffisants par la clientèle et si une autre ville desservie ne dispose pas d'atouts plus attractifs.

Enfin, la localisation des villes par rapport à la clientèle visée et par rapport aux autres villes est importante dans le potentiel de développement touristique. Le fait pour une capitale d'être desservie par TGV n'a pas toujours un rôle fondamental dès lors que les touristes qui la visitent sont étrangers et viennent en avion. En revanche, être une ville proche de la capitale et permettre à une clientèle internationale de faire un aller-retour en une ou deux journées devient un atout si ses actifs spécifiques lui permettent de se distinguer des autres villes. La localisation n'est donc pas seulement à considérer en elle-même mais aussi vis-à-vis des autres villes desservies et selon la provenance de la 
clientèle. Ainsi la clientèle francilienne et internationale de passage à Paris est fortement ciblée par les villes intermédiaires à une heure environ de Paris et proposant une bonne desserte de week-end, bien cadencée et adaptée à une arrivée en tout début de week-end et à un retour tardif le dimanche soir (Delaplace, Perrin, 2013).

Or la gouvernance peut influer sur ces différents éléments.

\section{B. Politique de valorisation touristique des dessertes TGV et efficacité de la Gouvernance}

Trois types de politiques de valorisation touristique sont mises en œuvre des dessertes TGV :

1 - La communication sur le positionnement de la ville par rapport à d'autres afin d'optimiser la perception qu'en a le touriste. Cette communication doit permettre d'identifier la ville comme possible destination (principale ou secondaire) et d'accroître le nombre de sites à visiter en jouant sur la complémentarité. Ainsi Arras a fait de sa proximité de Lille un atout. Un ciblage approprié de la clientèle et des lieux de diffusion facilite le changement d'image de ces villes en association avec une desserte TGV. De surcroît, ce changement d'image peut profiter aux villes sans que la desserte soit effectivement utilisée par les acteurs (Bazin et Delaplace, 2013).

2 - Les politiques d'accessibilité intra et inter urbaines. L'amélioration de l'intermodalité se traduit par la création de bretelles routières aux gares localisées sur LGV, l'offre de vélibs, de taxis, de tramway et de bus directement en sortie de gare. L'accessibilité aux villes secondaires peut passer par une irrigation des territoires, par dessertes TGV sur ligne classique, ou avec des « TERGV ».

3 - Le travail collaboratif entre acteurs pour proposer un panier de biens permettant d'attirer le touriste et le faire rester. Le recensement des actifs existants et une réflexion sur les nouveaux biens à proposer, susceptibles d'être complémentaires, permettent d'étoffer le panier de biens accessible aux touristes, à la fois pour rendre la destination attractive, mais aussi pour les faire rester et éviter la réduction de la durée des séjours. Les touristes sont en effet à la recherche d'un panier de biens leur permettant de « rentabiliser » la visite de la destination et de diversifier les lieux visités et les types de visites (musées, circuits pédestres à thème dans la ville, évènementiel, escapade proche pour une visite des sites naturels, etc.).

De nouveaux modes de coordination peuvent alors apparaitre entre:

1. territoires concurrents (pour une offre et une communication communes),

2. les collectivités et l'opérateur ferroviaire (pour la localisation des gares et leur cofinancement, le maintien de la desserte et l'adaptation des horaires au tourisme urbain et d'affaire, ou pour une communication commune),

3. l'office de tourisme et des prestataires privés (pour développer des produits innovants),

4. l'office et les hôtels (pour proposer les Packages),

5. le Centre des Congrès et les hôtels (afin d'assurer une offre d'hébergement suffisante).

47 A partir d'une cinquantaine d'entretiens réalisés avec des acteurs du tourisme (offices de tourisme, agences de développement économique, etc.) en France dans des villes de taille différente : des grandes villes (Nantes et Lille), des villes intermédiaires (Tours, Le Mans, Reims et Metz), et des villes petites et moyennes (Auray, Saverne, Charleville- 
Mézières, Arras, et Vendôme), le tableau 1 synthétise les outils de la gouvernance ainsi que son efficacité dans la valorisation touristique des villes TGV, par taille de ville.

Tableau 1 : Outils et efficacité de la gouvernance dans la valorisation touristique des villes TGV, par taille de ville

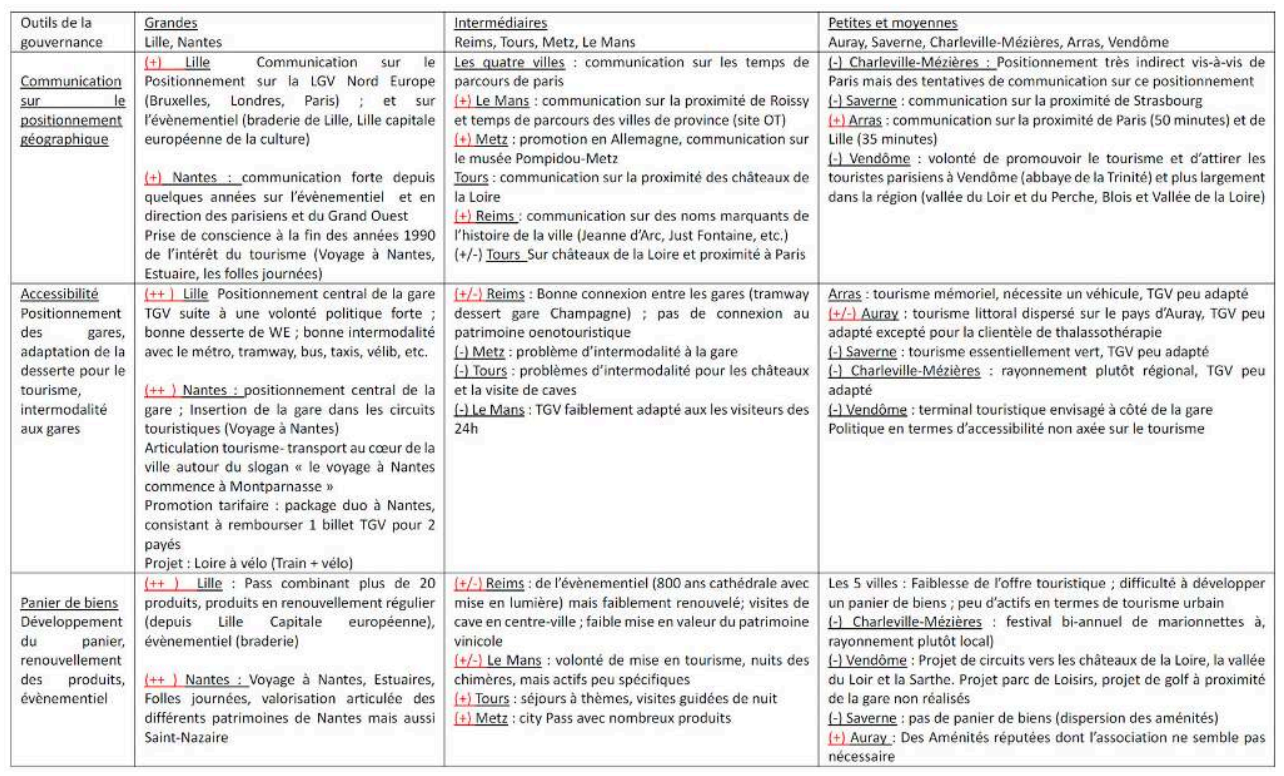

Source : les auteurs à l'aide d'entretiens auprès des acteurs du tourisme, et de Bazin et al., 2013b

En termes de positionnement: un certain nombre de villes, de grande taille, relativement bien positionnées par rapport à Paris et au réseau à grande vitesse européen, bénéficiant d'un patrimoine déjà connu, et dotées d'une desserte bien cadencée, tirent profit du TGV dans le domaine du tourisme. C'est le cas de Lille, à une heure de Paris en TGV, avec une bonne desserte en semaine pour le tourisme d'affaire, et le week-end pour le tourisme urbain. Lille est à 30 minutes de Bruxelles et à $1 \mathrm{~h} 30$ de Londres en TGV, ce qui permet de cibler une clientèle internationale résidant dans ces villes ou en séjour dans ces villes. A 50 minutes de la gare TGV Roissy Charles de Gaulle, Lille peut également capter une clientèle étrangère en visite à Paris. Certaines villes de taille intermédiaire, parviennent aussi à valoriser ce positionnement notamment lorsqu'elles se situent à moins d'une heure de Paris et proposent un patrimoine urbain important. Cela a été le cas du Mans qui a bénéficié d'une croissance de son tourisme d'affaires (Bazin et al., 2014). Si au-delà de ce patrimoine déjà connu un événementiel est associé, autour duquel une politique de communication forte est établie, la desserte TGV peut contribuer à favoriser la fréquentation touristique. Cela a été le cas de Reims en 2011 où la fréquentation touristique a connu un pic avec des festivités organisées autour des 800 ans de la cathédrale (cf. tableau 3). En revanche, l'évènementiel doit être renouvelé pour maintenir la tendance. En effet, à Reims si la ville y a gagné en termes d'image et semble avoir fidélisé la clientèle francilienne, la fréquentation est redescendue les années suivantes.

Les villes petites et moyennes ont toutefois beaucoup plus de difficultés à valoriser leur activité touristique avec le TGV, la desserte et les aménités y étant plus limitées. Cependant le TGV peut leur procurer un avantage en termes d'image. C'est le cas d'Arras (Bazin et al., 2013a) à 50 minutes de Paris en TGV. Si la ville dispose d'un bon potentiel en termes de tourismes urbain et mémoriel, est caractérisée par une volonté 
politique forte de renouvellement des produits, et une bonne desserte, les touristes n'y viennent que marginalement en TGV. La hausse sensible de la fréquentation touristique depuis 10 ans est davantage liée à un effet indirect d'image du TGV, associé à une valorisation des places, et à une communication sur la proximité avec Lille pour valoriser la destination. Charleville-Mézières, qui communique sur sa relation avec Paris en TGV en $1 \mathrm{~h} 30$ a vu de son côté sa fréquentation diminuer entre 2005 et 2010 , malgré un potentiel en termes de tourisme urbain et évènementiel. Les cas d'Auray (petite ville à proximité de Lorient et à $3 \mathrm{~h} 30$ en TGV de Paris) et de Saverne (tourisme historique et tourisme vert à 2 h00 de Paris et à proximité de Strasbourg) montrent à la fois la difficulté pour les petites villes d'utiliser le TGV à des fins de tourisme, mais également l'inadéquation entre un patrimoine touristique dispersé et l'usage du TGV.

En termes d'accessibilité : la plupart des villes desservies cherchent à améliorer l'offre intermodale en sortie de gare. Une des premières innovations a été de rapprocher les gares TGV et les gares centrales. Cela a été le cas à Lille, avec l'obtention d'une gare TGV en centre-ville suite à un accord intervenu entre la ville, la région et la SNCF pour la prise en charge financière du surcoût. Cela a aussi été le cas dans d'autres villes qui ont tiré les leçons des difficultés liées aux gares TGV excentrées. Mais toutes ne l'ont pas fait. Ainsi en raison de conflits entre Metz et Nancy, la gare Lorraine a été implantée entre les deux, et de surcroît n'est pas reliée au TER.

51 A Reims, le tramway mis en service peu de temps après la LGV-Est, ne devait initialement pas rejoindre la gare Champagne-Ardenne. Mais les collectivités locales ont fait en sorte que ce soit le cas et une volonté politique forte a également permis de relier la gare sur LGV à la gare centrale par TER en 10 minutes, et en tramway en 20 minutes. La clientèle touristique peut ainsi rejoindre facilement un hôtel en centreville ou directement les sites touristiques urbains. Cela permet également d'accéder aux territoires connectés par TER (les Ardennes notamment). Mais cette volonté d'irriguer des territoires par les TGV sur lignes classiques, comme c'est le cas de la LGV-Est vers les Ardennes, ou de la LGV Nord vers le littoral, si elle suscite des attentes en matière de tourisme, a en réalité peu d'impact. La ville de Sedan, par exemple a nourri des attentes concernant la hausse de fréquentation de son château réhabilité, alors que la fréquentation touristique est restée essentiellement régionale ou frontalière. Les villes petites et moyennes ne bénéficient donc que marginalement de la desserte TGV en matière de tourisme, ce qui conduit à discuter de la pertinence du prolongement des dessertes TGV sur ligne classique.

Dans de nombreux cas des politiques visent à faciliter cette intermodalité aux gares en adaptant l'offre de bus, de taxis ou la location de voiture, etc. Dans d'autres, ce sont des acteurs privés qui peuvent prendre en charge la jonction entre la gare et la destination finale (centre de thalassothérapie de Carnac). Dans d'autres enfin, l'intermodalité n'est absolument pas pensée. Ainsi à Tours, la connexion entre la gare et les Châteaux n'a pas été pensée (Troin, 2012). Parfois la signalétique est revue avec par exemple des bornes interactives en gare (combinant offre touristique et transport) ou une signalisation combinée gare/cœur de ville. 
Figure 3 : Borne interactive en gare de Reims

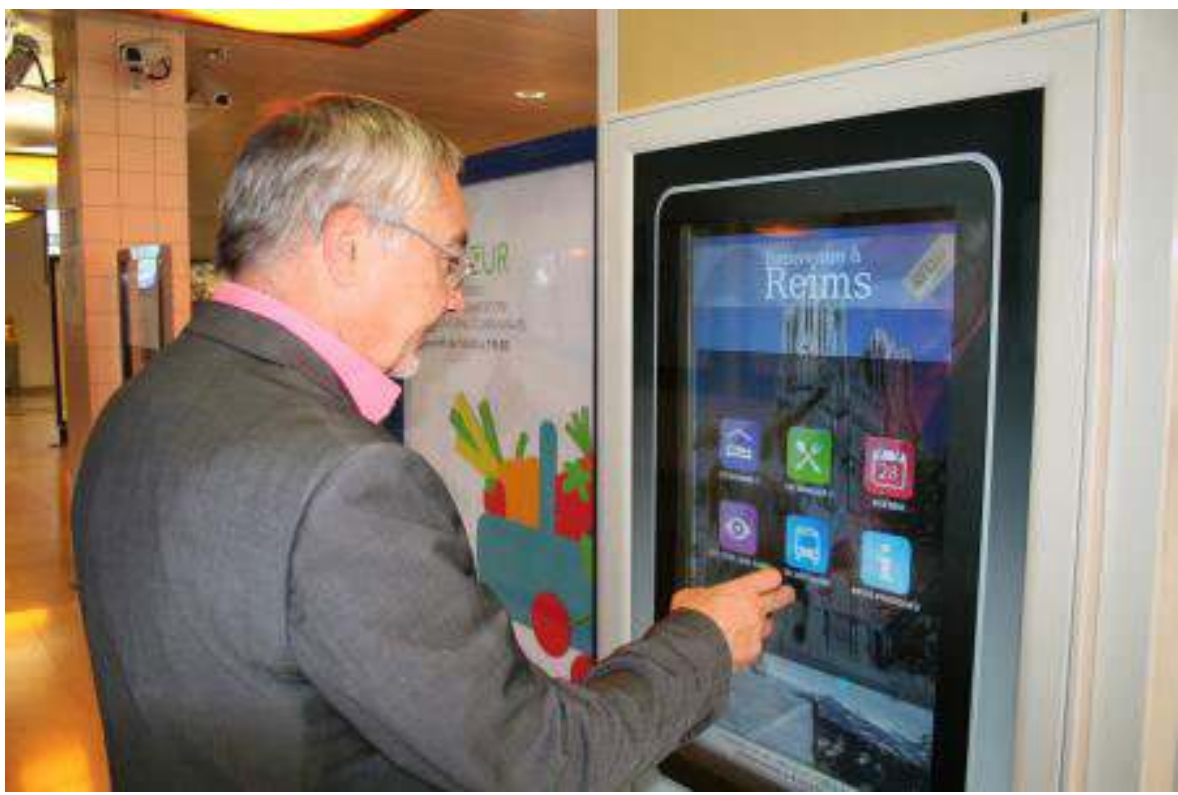

Figure 4 : Signalétique combinée gare/cœur de ville à Metz

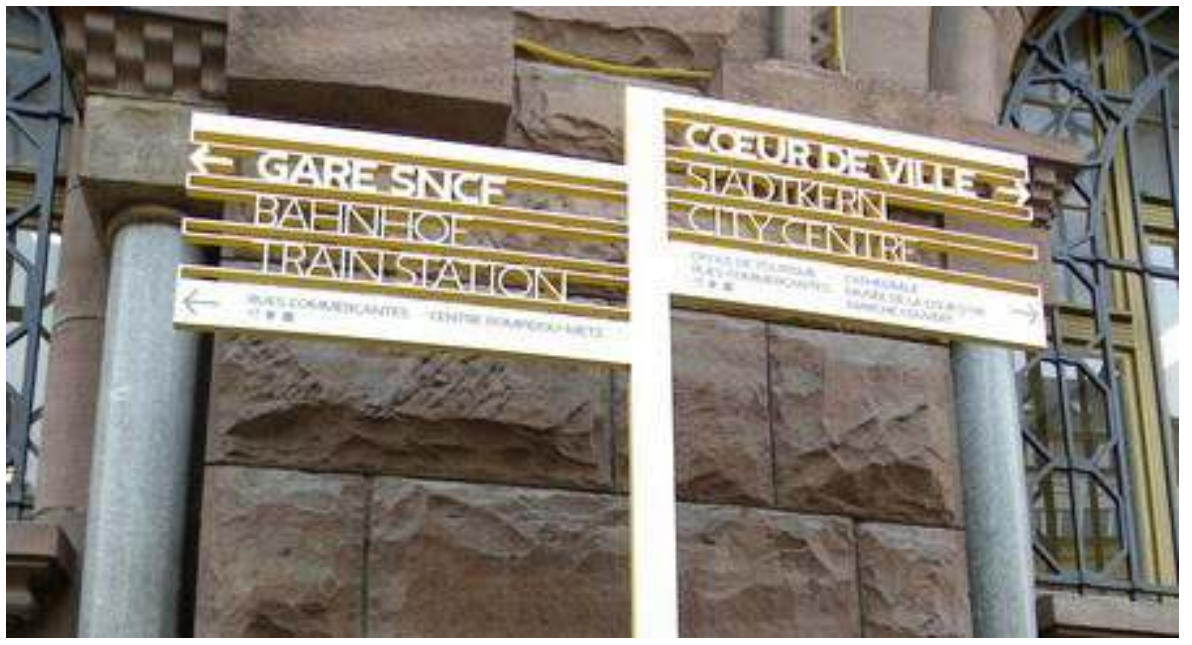

En revanche, la réflexion sur les cheminements piétons est le plus souvent absente (Coronado and al., 2014). Mais cette présence d'une offre intermodale de qualité ne suffit pas à développer le tourisme. Elle ne fait que conforter le service apporté aux touristes, surtout dans les grandes villes et villes intermédiaires dès lors qu'un travail est engagé sur la construction d'un panier de biens.

54 Concernant l'offre de paniers de biens : la concurrence entre villes desservies les amène à réfléchir aux aménités proposées aux touristes et à les présenter sous forme de de « packages » ciblant différents types de budgets. Cela conduit les collectivités locales et les opérateurs du tourisme à travailler ensemble pour fournir une tarification commune, sous forme par exemple de "pass " permettant l'accès à ces paniers de biens. Les OT cherchent dans certaines villes à coordonner l'offre de façon à proposer un « guichet unique» évitant une dispersion de l'offre et une perte de clientèle. Certains OT sont amenés à proposer des services d'opérateurs privés, complémentaires à l'offre classique orientée vers les musées, les visites guidées de monuments ou de villes. Les 
villes cherchent à multiplier les manifestations évènementielles de façon à attirer davantage les touristes urbains qui se déplacent en TGV. C'est le cas par exemple au Mans où la ville a proposé des spectacles nocturnes, ou encore à Reims où l'éventail de produits accessibles aux touristes urbains a été développé, comme des circuits à thèmes, ou des produits combinés Cathédrale/Champagne. Les destinations cherchent à se vendre également davantage aux prescripteurs du tourisme d'affaire, tourisme qui valorise la réduction des temps de parcours. Les villes espèrent ainsi entrer dans la «short list» des villes potentielles de Congrès ou de séminaire.

La valorisation des actifs liés au tourisme semble cependant profiter davantage aux villes bénéficiant d'un patrimoine spécifique et connu, surtout dans les grandes villes ou dans les villes intermédiaires. Le Mans, par exemple, a cherché à développer une offre spécifique avec la «nuit des chimères » et de nombreuses formules week-end en ciblant le marché francilien, d'autres grandes villes en province, ou encore l'étranger. Malgré cet effort de valorisation, le patrimoine touristique urbain autour du centre historique, quoiqu'indéniable, semble insuffisamment connu pour véritablement renforcer la fréquentation touristique et faire rester les touristes.

L'analyse des expériences de ces villes en matière de tourisme lié au TGV montre en particulier qu'il valorise surtout des lieux attirant certaines catégories de touristes, à revenus plutôt élevés, ayant des attentes fortes en matière d'offre touristique spécifique déjà connue et centrale. Des politiques de communication et une bonne desserte peuvent accompagner l'attractivité de ces villes mais ne la créent pas. En outre, le tourisme et notamment le tourisme d'affaires est affecté par la conjoncture. Une conjoncture dégradée peut alors remettre en question la possible valorisation de la desserte en matière de tourisme, même avec une gouvernance efficace.

\section{Conclusion}

57 La gouvernance locale autour des dessertes TGV mobilise de nombreux acteurs qui se coordonnent de différentes manières selon les territoires et selon les types de concurrence ou compromis préalables à la mise en service des dessertes TGV, lors des choix de tracés et de localisation des gares. Dans le domaine du tourisme, en raison des nombreuses attentes, un certain nombre de politiques se mettent en place. Celles-ci n'ont toutefois pas forcément les résultats escomptés, notamment dans les petites et moyennes villes qui n'offrent pas suffisamment d'aménités touristiques.

Dans le cas français, des politiques de communication, de renforcement de l'intermodalité, d'offre de paniers de biens et de services hôteliers et de restauration adaptés sont mises en œuvre mais sont surtout efficaces dans les grandes villes et les villes intermédiaires dotées d'un potentiel touristique important et diversifié. La coordination entre villes pour l'offre de paniers de biens permet d'éviter la réduction de la durée des séjours souvent consécutive à la mise en services des dessertes TGV. Toutefois les rabattements des dessertes TGV vers les villes petites et moyennes profitent rarement au tourisme, le tourisme de court séjour étant peu adapté aux multi-destinations, sauf pour les grandes villes proches de Paris.

Enfin le tourisme n'étant pas épargné en cas de contexte économique dépressif, y compris pour les grandes villes et villes intermédiaires qui remplissent ces conditions, 
les dessertes TGV ne sont pas une assurance contre ce risque, alors que les politiques de mise en tourisme mobilisent souvent des fonds importants.

1. Ces ressources présentent la particularité de n'être pas transférables à un autre territoire (Colletis, Pecqueur, 2004, Pecqueur, 2001) et de contribuer à l'existence d'avantages comparatifs en matière d'offre touristique.

\section{BIBLIOGRAPHY}

Amiard D., 1997, « Le tourisme d'affaire et de Congrès dans l'agglomération mancelle ", in Chevalier J. (1997), Le Mans 6 ans après l'arrivée du TGV, groupe de recherche en géographie sociale, ESO - Espaces géographiques et Sociétés, Université du Maine, Le Mans, 70p.

Bazin S., Beckerich C., Delaplace M., 2010, « Grande vitesse, activation des ressources spécifiques et développement du tourisme urbain : le cas de l'agglomération rémoise », Belgeo, 1-2, p.65-78.

Bazin S., Beckerich C., Blanquart C., Delaplace M., Vandenbossche L., 2011a « Grande vitesse ferroviaire et développement économique local : une revue de la littérature ». Recherche, Transports et Sécurité. 27-106, p.215-238.

Bazin S., Beckerich C., Delaplace M., 2011b, “ High Speed Railway, Service Innovations and Urban and Business Tourisms Development ", Chapitre 4 Dans SARMENTO Manuela, \& Alvaro MATIAS Economics and Management of Tourism: Trends and Recent Developments. Collecçao Manuais, Universidade Luisiada Editora, Lisboa, Portugal, 422p.

Bazin, S., Beckerich C., Delaplace M., 2013a, « Desserte TGV et villes petites et moyennes, Une illustration par le cas du tourisme à Arras, Auray, Charleville-Mézières et Saverne ", Les Cahiers Scientifiques du Transport, 63, p.33-62.

Bazin S., Beckerich C., Blanquart C., Delaplace M., 2013b, Les enjeux et opportunités des dessertes ferroviaires à grande vitesse en matière de développement local et de développement durable, rapport final, Contrat PREDIT, financement ADEME, mai, 186p.

Bazin S. et Delaplace M., 2013, « Desserte ferroviaire à grande vitesse et tourisme : entre accessibilité, image et outil de coordination », Teoros, vol. 32, $\mathrm{N}^{\circ} 2$, p.37-46.

Bazin, S., Beckerich C., Delaplace M., 2014, «Valorisation touristique du patrimoine et dessertes TGV dans les villes intermédiaires à moins d'1h30 de Paris : les cas de Reims, Metz, Le Mans et Tours ». Revue d'économie Régionale et Urbaine, n 5, p. 865-883.

Carrouet G., 2013, Du TGV Rhin-Rhône au territoire Rhin-Rhône : réticularité, mobilité et territorialité dans un espace intermédiaire. Thèse de doctorat en géographie-Aménagement, Université de Dijon, $518 \mathrm{p}$.

Coach Omnium, 2014, Etude sur l'évolution de la demande sur le marché du Tourisme d'affaires (MICE) Communiqué de presse -2 janvier

Colletis G., Pecqueur B., 2004, « Révélation des ressources spécifiques et coordination située », Economies et Institutions, ${ }^{\circ} 6-7, \mathrm{p} .51-74$. 
Coronado J-M., Garmendia M., Moyano A., 2014, HSR stations' missing link: Assessing the quality of pedestrian routes for tourism trips, 2nd International seminar "High Speed Rail Service and urban dynamics: Case studies in Europe" finance par le LabEX Futurs Urbains de Paris-Est, Naples, 3-4 Mars Czernek K., 2013, Determinants of cooperation in a tourist region, Annals of Tourism Research, Vol. 40, p.83-104.

Delaplace M., Perrin J., 2013, « Multiplication des dessertes TGV et Tourismes urbains et d'affaires, Regards croisés sur la Province et l'Ile de France », Recherche Transport et Sécurité, Vol. 29, p.177-191.

ISIS, 2004, Analyse de l'impact du TGV-Est sur les agglomérations de Metz, Nancy, Epinal et Thionville. Rapport de phase 2, ADIELOR.

Jacquier C., 2008, Gouvernance, la pluralité des acceptions, communication au Colloque « Vers une nouvelle gouvernance des territoires », Reims, 15 septembre.

Jones M. F., Singh N. and Hsiung Y., 2013, "Determining the Critical Success Factors of the Wine Tourism Region of Napa from a Supply Perspective", International Journal of Tourism Research, online.

Kamel K., Matthewman R., 2008, The non-transport impacts of High-Speed Trains on regional economic development : a review of the literature, Locate in Kent, consulté le 30 novembre 2013, URL : http:// www.locateinkent.com/images/assets/High\%20Speed\%20Train\%20Report\%202008.pdf

Leloup F., Moyart L., Pecqueur B., 2005, « La gouvernance territoriale comme nouveau mode de coordination territoriale?", Géographie, Economie, Société, vol. 7, p.321-331.

Ménerault P., 1997, Processus de territorialisation de la grande vitesse ferroviaire: le TGV et les régions: Le cas du Nord-Pas de Calais. INRETS, 102p.

Mignerey P., 2013, Les effets territoriaux de la grande vitesse. La Documentation Française, 96 p. Mollard A., Pecqueur B., 2007, « De l'hypothèse au modèle du panier de biens et de service : histoire succincte d'une recherche », Economie Rurale, $\mathrm{n}^{\circ}$ 300, p.110-114.

Pagliara F., Vassalo J.M., Roman C., 2012, "High Speed Rail versus air transportation. Case study of Madrid-Barcelona, Spain”, Transportation Research Record, 2289, p.10-17.

Pagliara F., Delaplace M. and Vassalo J.M., 2014, High Speed trains and tourists: what is the link? Evidence from the French and the Spanish capitals. Proceedings of the conference Urban Transport XX Urban Transport and the Environment in the 21st century. WIT Transactions on the Built Environment, $\mathrm{n}^{\circ}$ 138, p.17-27.

Pecqueur B., 2001, « Qualité et Développement territorial : l'hypothèse du panier de biens et de services territorialises ", Economie Rurale n²61, p.37-49.

Prideaux B., 2000, “The role of the transport system in destination development", Tourism Management $n^{\circ} 21$, p. 53-63.

SETEC ORGANISATION, 2005, Etude des effets de la réalisation d'une LGV entre Bordeaux et Narbonne sur le développement des aires urbaines d'Agen et de Montauban et sur l'aménagement des territoires traversés, pour Réseau Ferré de France, 20p.

Sèze B. de, 2008, Le tourisme d'affaires : Un enjeu pour la destination France, Cahier Espaces, $\mathrm{N}^{\circ}$ 97, pp. 10-16.

Troin J-F., 2012, TGV et fréquentation touristique : une image contrastée en Val de Loire, Colloque ASRDLF, France, Belfort 
Wang Y., Fesenmaier D. R., 2007, « Collaborative destination marketing: A case study of Elkhart county, Indiana", Tourism Management, $\mathrm{n}^{\circ} 28$, p. 863-875.

\section{ABSTRACTS}

In territories supplied by High speed rail services, the actors expect a dynamic economy, in general, and of the tourism in particular, especially when they are facing to strong difficulties. In order to transform this opportunity into a development tool, new kinds of cooperation can emerge. Some of them that can be called governance allowed served territories to benefit from High speed rail service and to grow their attractiveness. We will focus on a field, the tourism sector, in which expected benefits are high and the cooperation policies necessary. By focusing on French cities of different sizes, we will analyze the governance tools used in tourism field regarding to high speed rail services and their efficacity.

La mise en service d'une desserte TGV génère des attentes importantes dans les territoires desservis notamment dans le domaine du tourisme, en particulier lorsqu'ils connaissent des difficultés économiques. De façon à transformer cette opportunité en outil de développement, des formes de coopération nouvelles peuvent émerger. Certaines de ces formes de coopération innovantes, qualifiées de gouvernance ont permis aux territoires desservis de tirer profit de cette desserte et d'accroître leur attractivité. Nous nous focalisons ici sur un des domaines dans lesquels les espoirs de valorisation des dessertes TGV sont importants et les politiques de coopération mises en place autour des dessertes TGV fondamentales, le tourisme. A partir du cas de villes françaises de taille différente, nous analysons les outils de gouvernance mis en place dans le domaine du tourisme en lien avec les dessertes à grande vitesse et leur efficacité.

Die Inbetriebnahme einer TGV-Verkehrsanbindung verursacht erhebliche Wartezeiten in den jeweiligen Anfahrtsgebieten, insbesondere dann, wenn sie mit wirtschaftlichen Schwierigkeiten konfrontiert wird. Um diese Opportunität als Entwicklungsmittel zu optimieren, können neue Kooperationsformen entstehen. Einige dieser, zur "Gouvernance" ernannten und innovativen Kooperationsformen, haben den Anschlussgebieten ermöglicht, die Vorteile von einer solchen Verkehrsanbindung zu ziehen und ihre Attraktivität zu fördern. Unser Hauptaugenmerk legen wir hier auf einen der Bereiche, der große Hoffnung auf die Hochgeschwindigkeitszugvalorisation setzt und für den, die eingesetzte Kooperationspolitik bezüglich der TGV-Anbindungen, fundamental ist, der Tourismus. Anhand unterschiedlich großer französischer Städte analysieren wir die eingesetzten "Gouvernance" Mittel und deren Effektivität, die im Zusammenhang des Tourismusbereiches und der Hochgeschwindigkeitszugverbindungen stehen und eingesetzt werden.

\section{INDEX}

Mots-clés: coopération, dessertes TGV, gouvernance, tourisme, villes françaises

Keywords: cooperation, French cities, governance, high-speed rail, tourism

Schlüsselwörter: französischer Städte, Gouvernance, Kooperationspolitik, TGVVerkehrsanbindung, Tourismusbereich 


\section{AUTHORS}

\section{SYLVIE BAZIN-BENOIT}

Maître de conférences - Université de Reims Champagne-Ardenne - Laboratoire REGARDS - 57 bis, rue Pierre Taittinger 51096 Reims Cedex - sylvie.bazin@univ-reims.fr

\section{MARIE DELAPLACE}

Professeure - Université de Paris-Est Marne-la-Vallée - Ecole d'Urbanisme de Paris Lab'Urba - 5 Bd Descartes 77454 Champs sur marne - marie.delaplace@u-pem.fr 